\title{
Torsades De Pointes by ECG Finding
}

National Cancer Institute

\section{Source}

National Cancer Institute. Torsades De Pointes by ECG Finding. NCI Thesaurus. Code C50779.

An electrocardiographic finding of an atypical rapid polymorphic ventricular tachycardia with a characteristic rotation of the QRS complex around the isoelectric baseline, occurring in the setting of a prolonged QT interval. In addition, the QRS complex displays a periodic waxing and waning of amplitude on the electrocardiogram. 